\title{
Symmetry Properties and Solutions of Shallow Water Equations
}

\author{
Yu. A. Chirkunov ${ }^{1 * *}$, E. O. Pikmullina ${ }^{2}$ \\ ${ }^{1}$ Novosibirsk State Technical University, pr. K. Marksa 20, Novosibirsk, 630092, Russia \\ ${ }^{2}$ Institute of Computational Technologies, Siberian Branch, Russian Academy of Sciences,pr. Akademika Lavrentjeva 6, Novosibirsk, \\ 630090 Russia \\ *Corresponding Author: chr01@rambler.ru
}

Copyright (C) 2014 Horizon Research Publishing All rights reserved.

\begin{abstract}
Within one-dimensional model of shallow water it is investigated a one-parameter family of equations describes the propagation of surface waves above a straight bottom. The parameter of the family is the slope of the bottom. This family is generated by the equations of one-dimensional model of shallow water with a horizontal bottom. By means of the method of $A$-operators it is found that this system has an infinite aggregate of non-trivial zero-order conservation laws generated by the system of linear differential equations. As a result of special choice of the hodograph transformation, the system of equations of one-dimensional model of shallow water with a horizontal bottom is generated by the same system of linear differential equations. The group analysis of the systems is carried out. An infinite aggregate of non-degenerate solutions of the equations of one-dimensional model of shallow water with a straight bottom is received. All of the degenerate solutions of these equations are found. Thus, the data base of exact solutions of the equations of one-dimensional model of shallow water with a straight bottom is created. The solutions obtained in this paper may be used in the study of tsunami waves and fluid distribution in channels.
\end{abstract}

Keywords Shallow Water, Conservation Laws, The Method Of $A$-Operators, The Group Analysis Of Differential Equations, Partially Invariant Solutions, Invariant Solutions, Non-Degenerate Solutions, Degenerate Solutions

\section{Introduction}

One-dimensional shallow water model

$$
v_{\tau}+v v_{y}+\eta_{y}=0, \eta_{\tau}+((\eta+k y) v)_{y}=0
$$

is used to study tsunami waves and fluid distribution in channels [1-7]. Where: $v=v(\tau, y)$ is propagation velocity of surface waves with a free surface $z=\eta(\tau, y)+k y$ along axis oy above a straight bottom $z=k y, k=$ const is the slope of the bottom, $o y z$ is rectangular coordinate system, $\tau$ is time.

If $k=0$ then the system (1) describes the propagation of surface waves above a horizontal bottom and if $k \neq 0$ above a sloping bottom.

If $k \neq 0$, then after the change of variables

$$
t=k \tau, x=k\left(y-\frac{k \tau^{2}}{2}\right), v=u(t, x)+t, \eta=h(t, x)-x-\frac{t^{2}}{2}
$$

the system (1) is reduced to the system

$$
u_{t}+u u_{x}+h_{x}=0, h_{t}+u h_{x}+h u_{x}=0
$$

which is written for the variables $t, x, u, h(h \geq 0)$ by the system (1) with the slope of the bottom $k=0$. Therefore, due to the generation (2), one-parameter system family (1) with the parameter $k \in(-\infty, \infty)$ is generated by the system (1) with the parameter $k=0$, namely by the system (3) which is the only object during the study of one-dimensional model of shallow water with a horizontal bottom. 


\section{Conservation Laws for the System (3)}

The conservation law of zero-order for the system (3) is called [8,9] the type correlation made because of the equations of the system

$$
D_{t} A^{0}+D_{x} A^{1}=0
$$

where $A^{k}=A^{k}(t, x, u, h)(k=0 ; 1) ; D_{t}, D_{x}$ are the total differentiation operators in the variables $t, x$ respectively. The two equations of the system (3) are the conservation laws of zero-order. To find all nontrivial conservation laws of zero-order, is used the method of $A$-operators proposed by Yu. A. Chirkunov [9-13]. The conservation law

$$
A^{0}=h, A^{1}=u h
$$

is taken as a generating one. It is defined by the second equation of the system (3).

From the system of constitutive equations obtained from the correlation

$$
\left.\left(X\left(h_{t}+u h_{x}+h u_{x}\right)\right)\right|_{(3)}=0,
$$

where $A$-operator $X=\xi^{1}(t, x, u, h) \partial_{u}+\xi^{2}(t, x, u, h) \partial_{h}+\ldots$ is an evolutionary operator of generalized symmetry [8-13] follows, that the factor set of zero-order $A$-operators for the system (3) by the range of its trivial $A$-operators is generated by the operators:

$$
X=\frac{f-u g}{h} \partial_{u}+g \partial_{h}+\ldots,
$$

where function $g=g(u, h)$ is a solution of an equation

$$
g_{u u}=h g_{h h},
$$

and function $f=f(u, h)$ is determined from the integrable system

$$
f_{u}=u g_{u}+h g_{h}, f_{h}=g_{u}+u g_{h}
$$

The operator rule (6) for the components of the conservation law assumed by the formulae (5), generates the components $A^{0}, A^{1}$ of all nontrivial conservation laws of zero-order for the system (3). Precisely:

$$
A^{0}=g(u, h), A^{1}=f(u, h) .
$$

After the substitution

$$
g_{u}=h \Phi(u, h), g_{h}=-\Psi(u, h)
$$

the equation (7) changes to

$$
\Phi_{u}+\Psi_{h}=0
$$

and the system (8) changes to

$$
f_{u}=h(u \Phi-\Psi), f_{h}=h \Phi-u \Psi .
$$

The condition of consistency of correlations (10) generates the equation

$$
\Psi_{u}+(h \Phi)_{h}=0 .
$$

Thus, the system (3) has an infinite aggregate of non-trivial zero-order conservation laws determined by the correlation (4), where

$$
\begin{aligned}
A^{0} & =\int h \Phi(u, h) d u-\Psi(u, h) d h \\
A^{1} & =\int h(u \Phi(u, h)-\Psi(u, h)) d u+(h \Phi(u, h)-u \Psi(u, h)) d h
\end{aligned}
$$

where $(\Phi(u, h), \Psi(u, h))$ is a solution of the system of linear equations 


$$
\Phi_{u}+\Psi_{h}=0, \Psi_{u}+(h \Phi)_{h}=0
$$

\section{Conservation Laws for the System (1).}

Let $k \neq 0$. Direct calculations determine that for any pair of functions $\left(A^{0}(t, x, u, h), A^{1}(t, x, u, h)\right)$ the identity is realized after the change (2)

$$
k\left(D_{t} A^{0}+D_{x} A^{1}\right)=D_{\tau} A^{0}+D_{y}\left(A^{1}+k \tau A^{0}\right) .
$$

Hence, if $\left(A^{0}(t, x, u, h), A^{1}(t, x, u, h)\right)$ are the components of an arbitrary zero-order conservation law (4) for the system (3), then the functions

$$
\begin{gathered}
B^{0}=A^{0}\left(k \tau, k\left(y-\frac{k \tau^{2}}{2}\right), v-k \tau, \eta+k y\right) \\
B^{1}=A^{1}\left(k \tau, k\left(y-\frac{k \tau^{2}}{2}\right), v-k \tau, \eta+k y\right)+k \tau A^{0}\left(k \tau, k\left(y-\frac{k \tau^{2}}{2}\right), v-k \tau, \eta+k y\right)
\end{gathered}
$$

comply with the correlation due to the system equations (1)

$$
D_{\tau} B^{0}+D_{y} B^{1}=0
$$

i.e. they are the components of the zero-order conservation law for the system (1).

Conversely, if $\left(B^{0}(\tau, y, v, \eta), B^{1}(\tau, y, v, \eta)\right)$ are the components of an arbitrary zero-order conservation law (14) for the system (1) where $k \neq 0$, then the functions are the components of the zero-order conservation law (4) for the system (3).

$$
\begin{gathered}
A^{0}=B^{0}\left(k \tau, k\left(y-\frac{k \tau^{2}}{2}\right), v-k \tau, \eta+k y\right), \\
A^{1}=B^{1}\left(\frac{t}{k}, \frac{1}{k}\left(x+\frac{t^{2}}{2}\right), u+t, h-x-\frac{t^{2}}{2}\right)-t B^{0}\left(\frac{t}{k}, \frac{1}{k}\left(x+\frac{t^{2}}{2}\right), u+t, h-x-\frac{t^{2}}{2}\right)
\end{gathered}
$$

then the functions are the components of the zero-order conservation law (4) for the system (3).

In this way, the components of all zero-order conservation laws (14) for the system (1) are determined by the formulae (11)-(13).

\section{The Symmetry Properties of the System (3)}

The operator admitted by the system (3) is sought in the form

$$
\xi_{0}(t, x, u, h) \partial_{t}+\xi_{1}(t, x, u, h) \partial_{x}+\sigma_{1}(t, x, u, h) \partial_{u}+\sigma_{2}(t, x, u, h) \partial_{h} .
$$

The condition of invariance with respect to this operator of manifold, determined by the system (3) after the splittance according to the parameter derivatives, generates the system of constitutive equations which ends in involution and integrates. As a result of it, the system (3) admits a pseudogroup of Lie transformations generated by the operators

$$
\begin{gathered}
X_{1}=t \partial_{t}+x \partial_{x}, X_{2}=t \partial_{x}+\partial_{u}, X_{3}=t \partial_{t}-u \partial_{u}-2 h \partial_{h}, \\
Z=\Phi(u, h) \partial_{t}+(\Psi(u, h)+u \Phi(u, h)) \partial_{x},
\end{gathered}
$$

where $(\Phi=\Phi(u, h), \Psi=\Psi(u, h))$ is any solution of the system (12).

Thus, the set of zero-order conservation laws for the system (3) and its main group of Lie transformations are determined by the same system of linear equations (12).

On the strength of Lie theorem [9], the formulae of solution production ("birth") are generated from the symmetry property (14). If $u=u(t, x), h=h(t, x)$ is the solution of the system (3), then the solution of the system (3) written for the 
variables $t^{\prime}, x^{\prime}, u^{\prime}, h^{\prime}$ is the following pairs of functions $\left(u^{\prime}\left(t^{\prime}, x^{\prime}\right), h^{\prime}\left(t^{\prime}, x^{\prime}\right)\right)$ :

$$
\begin{gathered}
u^{\prime}=a_{3}\left(a_{2}+u\left(a_{1} a_{3} t^{\prime}+a_{4}, a_{1}\left(x^{\prime}-a_{2} a_{3} t^{\prime}\right)+a_{5}\right)\right), \\
h^{\prime}=a_{3}^{2} h\left(a_{1} a_{3} t^{\prime}+a_{4}, a_{1}\left(x^{\prime}-a_{2} a_{3} t^{\prime}\right)+a_{5}\right) \\
u^{\prime}=u\left(t^{\prime}-\Phi\left(u^{\prime}, h^{\prime}\right), x^{\prime}-\Psi\left(u^{\prime}, h^{\prime}\right)-u \Phi\left(u^{\prime}, h^{\prime}\right)\right) \\
h^{\prime}=h\left(t^{\prime}-\Phi\left(u^{\prime}, h^{\prime}\right), x^{\prime}-\Psi\left(u^{\prime}, h^{\prime}\right)-u \Phi\left(u^{\prime}, h^{\prime}\right)\right)
\end{gathered}
$$

Where $a_{1}, a_{2}, a_{3}, a_{4}, a_{5}\left(a_{1} a_{3} \neq 0\right)$ are the arbitrary real constants and $\Phi=\Phi(u, h), \Psi=\Psi(u, h)$, is any solution of the system (12).

The formulae of solution production of the system (1) where $k \neq 0$ are generated on the strength of the transformation (2). In particular, if $v=v(\tau, y), \eta=\eta(\tau, y)$ is the solution of the system (1), then the following pairs of functions $v^{\prime}=v^{\prime}\left(\tau^{\prime}, y^{\prime}\right), \eta^{\prime}=\eta^{\prime}\left(\tau^{\prime}, y^{\prime}\right)$ are the solution of the system (1) written for the variables $\tau^{\prime}, y^{\prime}, v^{\prime}, \eta^{\prime}$.

$$
\begin{aligned}
& v^{\prime}=v\left(a_{1} k \tau^{\prime}, a_{1} k y^{\prime}+\frac{a_{1}}{2}\left(a_{1}-1\right)\left(k \tau^{\prime}\right)^{2}\right)-\left(a_{1}-1\right) k \tau^{\prime}, \\
& \eta^{\prime}=\eta\left(a_{1} k \tau^{\prime}, a_{1} k y^{\prime}+\frac{a_{1}}{2}\left(a_{1}-1\right)\left(k \tau^{\prime}\right)^{2}\right)+\frac{1}{2}\left(a_{1}-1\right)\left(2 k y^{\prime}+a_{1}\left(k \tau^{\prime}\right)^{2}\right) \text {, } \\
& v^{\prime}=v\left(k \tau^{\prime}, k\left(y^{\prime}-a_{2} \tau^{\prime}\right)\right)+a_{2}, \eta^{\prime}=\eta\left(k \tau^{\prime}, k\left(y^{\prime}-a_{2} \tau^{\prime}\right)\right)-a_{2} k \tau^{\prime} \\
& v^{\prime}=a_{3}\left(v\left(a_{3} k \tau^{\prime}, k y^{\prime}+\frac{\left(k \tau^{\prime}\right)^{2}}{2}\left(a_{3}^{2}-1\right)\right)-a_{3} k \tau^{\prime}\right)+k \tau^{\prime}, \\
& \eta^{\prime}=a_{3}^{2}\left(\eta\left(a_{3} k \tau^{\prime}, k y^{\prime}+\frac{\left(k \tau^{\prime}\right)^{2}}{2}\left(a_{3}^{2}-1\right)\right)+k y^{\prime}+\frac{\left(k \tau^{\prime}\right)^{2}}{2}\left(a_{3}^{2}-1\right)\right)-k y^{\prime} \\
& v^{\prime}=v\left(k \tau^{\prime}+a_{4}, k\left(y^{\prime}+a_{4} \tau^{\prime}\right)+\frac{a_{4}^{2}}{2}+a_{5}\right)+a_{4}, \\
& \eta^{\prime}=\eta\left(k \tau^{\prime}+a_{4}, k\left(y^{\prime}+a_{4} \tau^{\prime}\right)+\frac{a_{4}^{2}}{2}+a_{5}\right)+a_{4} k \tau^{\prime}+\frac{a_{4}^{2}}{2}+a_{5} ; \\
& v=k \tau-\Phi(v-k \tau, \eta+k y)+v^{\prime}(\Phi(v-k \tau, \eta+k y), \Psi(v-k \tau, \eta+k y)+\lambda(\tau, y, k, v, \eta, \Phi)), \\
& \eta=\Psi(v-k \tau, \eta+k y)-k y+\lambda(\tau, y, k, v, \eta, \Phi)+ \\
& +\eta^{\prime}(\Phi(v-k \tau, \eta+k y), \Psi(v-k \tau, \eta+k y)+\lambda(\tau, y, k, v, \eta, \Phi)) \\
& \left(\lambda(\tau, y, k, v, \eta, \Phi)=(v-k \tau) \Phi(v-k \tau, \eta+k y)+\frac{\Phi^{2}(v-k \tau, \eta+k y)}{2}\right) .
\end{aligned}
$$

\section{Non-degenerate Solutions}

The solution $u=u(t, x), h=h(t, x)$ of the system (3) will be called a non-degenerate one in case it is different from zero Jacobian $\frac{\partial(u, h)}{\partial(t, x)} \neq 0$. Analogously, solution $v=v(\tau, y), \eta=\eta(\tau, y)$ of the system (1) will be called a non-degenerate one in case it is different from zero Jacobian $\frac{\partial(v, \eta)}{\partial(\tau, y)} \neq 0$. The sets of non-degenerate solutions of the systems (1) and (3) are linked by the reversible transformation (2). 
By the set of non-degenerate solutions the system (3) becomes linear with the help of hodograph transformation, which is convenient to choose in accordance with the conservation laws (11) of the system (3) and its symmetry property (15):

$$
t=\Phi(u, h), x=\Psi(u, h)+u \Phi(u, h),
$$

As the result of this transformation, the system (3) is equated with the linear system (12).

The solutions of the system (3) and (12) are linked in the following way.

Any non-degenerate solution $(u(t, x), h(t, x))$, of the system (3) determines a non-degenerate solution of the system (12) by the formulae (23), i.e. the solution $(\Phi(u, h), \Psi(u, h))$ complies with the condition $\frac{\partial(t, x)}{\partial(u, h)} \neq 0$. On the strength of (23) and (12), it means that $\left(\Phi_{u}^{2}+h \Phi_{u}^{2}\right) \neq 0$, i.e. any solution of the system (12) with $\Phi \neq$ const is a non-degenerate one. All of the degenerate solutions of the system are as follows

$$
\Phi=c, \Psi=-c u,
$$

where $c$ is an arbitrary real constant.

Conversely, any non-degenerate solution $(\Phi(u, h), \Psi(u, h))$ of the system (12) which complies with the condition (24) in some variables' part $u, h$, tacitly determines non-degenerate solution $(u(t, x), h(t, x))$ of the system (3) in the corresponding domain of variables $t, x$.

Thus, the system of linear differential equations (12) determines for the system of equations (3) of one-dimensional model of shallow water with a horizontal bottom, the range of zero-order conservation laws, its main group of Lie transformations, and its set of non-degenerate solutions. On the strength of the transformation (2), it is also true for the system (1) of the equations of one-dimensional model of shallow water with a sloping bottom. Next, the group analysis of the system (12) is carried out. The system's invariant and partially invariant solutions make it possible to generate zero-order conservation laws for the systems (1), (3) of the equations of one-dimensional model of shallow water with a straight bottom and their non-degenerate exact solutions.

\section{The Symmetry Properties of the System (12)}

The operator admitted by the system (12) is sought in the form

$$
\xi_{1}(u, h, \Phi, \Psi) \partial_{u}+\xi_{2}(u, h, \Phi, \Psi) \partial_{h}+\eta_{1}(u, h, \Phi, \Psi) \partial_{\Phi}+\eta_{2}(u, h, \Phi, \Psi) \partial_{\Psi}
$$

The condition of invariance with respect to this operator of manifold, determined by the equations (12) after the splittance according to the parameter derivatives, generates the system of constitutive equations which ends in involution and integrates after the third extension. As a result of it, the main group of Lie transformations of the system (12) (factor group by normal subgroup linked with the system linearity) is generated by the operators

$$
\begin{gathered}
X_{1}=\left(\frac{u^{2}}{4}+h\right) \partial_{u}+u h \partial_{h}+\left(\frac{\Psi}{2}-u \Phi\right) \partial_{\Phi}+\frac{1}{2}(h \Phi-u \Psi) \partial_{\Psi}, \\
X_{2}=u \partial_{u}+2 h \partial_{h}-\Phi \partial_{\Phi}, \quad X_{3}=\partial_{u}, \quad X_{4}=\Phi \partial_{\Phi}+\Psi \partial_{\Psi}
\end{gathered}
$$

On the strength of Lie theorem [9] and the system linearity (12), the following formulae of its solution production are generated by the symmetry property (25). It is relevant for any pair of complex-valued functions $(\Phi(u, h), \Psi(u, h))$ which are the solution of the system (12). The solution of the system (12) written for the variables $u^{\prime}, h^{\prime}, \Phi^{\prime}, \Psi^{\prime}$ is the following pairs of functions $\left(\Phi^{\prime}\left(u^{\prime}, h^{\prime}\right), \Psi^{\prime}\left(u^{\prime}, h^{\prime}\right)\right)$, defined by the formulae

$$
\begin{aligned}
& \Phi^{\prime}\left(u^{\prime}, h^{\prime}\right)=\operatorname{Re}\left\{\mu^{3}\left(u, h, b_{1}\right)\left(4 \Phi(u, h)+b_{1}(2 \Psi(u, h)-u \Phi(u, h))\right)\right\}, \\
& \Psi^{\prime}\left(u^{\prime}, h^{\prime}\right)=-\operatorname{Re}\left\{16 \mu\left(u, h, b_{1}\right)\left(4 \Psi(u, h)+b_{1}(2 h \Phi(u, h)-u \Psi(u, h))\right)\right\} ; \\
& \Phi^{\prime}\left(u^{\prime}, h^{\prime}\right)=\operatorname{Im}\left\{\mu^{3}\left(u, h, b_{1}\right)\left(4 \Phi(u, h)+b_{1}(2 \Psi(u, h)-u \Phi(u, h))\right)\right\}, \\
& \Psi^{\prime}\left(u^{\prime}, h^{\prime}\right)=-\operatorname{Im}\left\{16 \mu\left(u, h, b_{1}\right)\left(4 \Psi(u, h)+b_{1}(2 h \Phi(u, h)-u \Psi(u, h))\right)\right\} ;
\end{aligned}
$$




$$
\begin{gathered}
\Phi^{\prime}\left(u^{\prime}, h^{\prime}\right)=b_{2} \operatorname{Re} \Phi\left(b_{2}\left(u^{\prime}+b_{3}\right), b_{2}^{2} h^{\prime}\right), \Psi^{\prime}\left(u^{\prime}, h^{\prime}\right)=\operatorname{Re} \Psi\left(b_{2}\left(u^{\prime}+b_{3}\right), b_{2}^{2} h^{\prime}\right) \\
\Phi^{\prime}\left(u^{\prime}, h^{\prime}\right)=b_{2} \operatorname{Im} \Phi\left(b_{2}\left(u^{\prime}+b_{3}\right), b_{2}^{2} h^{\prime}\right), \Psi^{\prime}\left(u^{\prime}, h^{\prime}\right)=\operatorname{Im} \Psi\left(b_{2}\left(u^{\prime}+b_{3}\right), b_{2}^{2} h^{\prime}\right)
\end{gathered}
$$

where $\mu\left(u, h, b_{1}\right)=\left(b_{1}^{2} u^{2}-4 b_{1}^{2} h-8 b_{1} u+16\right)^{\frac{1}{2}} ; b_{1}, b_{2}, b_{3}, b_{4} \quad\left(b_{2} b_{4} \neq 0\right)$ are arbitrary real constants.

On the strength of the infinitesimal formula of solution production [9], the symmetry property (25) and the system linearity (12) generate the following formulae of its solution production. Precisely, if $(\Phi(u, h), \Psi(u, h))$ is an arbitrary smooth solution of the system (12) then the solutions of the system are the following pairs of functions $\left(\Phi^{\prime}\left(u^{\prime}, h^{\prime}\right), \Psi^{\prime}\left(u^{\prime}, h^{\prime}\right)\right)$ :

$$
\begin{array}{r}
\Phi^{\prime}(u, h)=\left(\frac{u^{2}}{2}+2 h\right) \Phi_{u}(u, h)+2 u h \Phi_{h}(u, h)-\Psi(u, h)+2 u \Phi(u, h) \\
\Psi^{\prime}(u, h)=\left(\frac{u^{2}}{2}+2 h\right) \Psi_{u}(u, h)+2 u h \Psi_{h}(u, h)-h \Phi(u, h)+u \Psi(u, h) \\
\Phi^{\prime}(u, h)=u \Phi_{u}(u, h)+2 h \Phi_{h}(u, h)+\Phi(u, h), \Psi^{\prime}(u, h)=u \Psi_{u}(u, h)+2 h \Psi_{h}(u, h) ; \\
\Phi^{\prime}(u, h)=\Phi_{u}(u, h), \Psi^{\prime}(u, h)=\Psi_{u}(u, h) .
\end{array}
$$

Owing to the system linearity (12), any linear combination of solutions of this system is its solution.

\section{Exact Solutions of the System (12)}

To classify the invariant and partially invariant solutions of the system (12), the optimal system of dissimilar Lie subalgebras with the basis (25) is set up. Operator $X_{4}$ is its center. Each subalgebra of this optimal system of subalgebras corresponds with a subgroup of the main group of the system (12) generated by it. As a result, we have an optimal system of subgroups of the main group of the system. It contains four one-parameter subgroups $\left\langle X_{4}\right\rangle,\left\langle X_{2}+\alpha X_{4}\right\rangle,\left\langle X_{3}+\beta X_{4}\right\rangle$, $\left\langle X_{1}+X_{3}+\varepsilon X_{4}\right\rangle$ six two-parameter subgroups $\left\langle X_{2}, X_{3}\right\rangle,\left\langle X_{2}+\gamma X_{3}, X_{4}\right\rangle,\left\langle X_{2}+X_{4}, X_{3}\right\rangle,\left\langle X_{1}, X_{4}-X_{2}\right\rangle$, $\left\langle X_{1}+\delta X_{3}, X_{4}\right\rangle,\left\langle X_{2}+\sigma X_{4}, X_{1}\right\rangle$, and three three-parameter subgroups $\left\langle X_{1}, X_{2}, X_{4}\right\rangle,\left\langle X_{2}, X_{3}, X_{4}\right\rangle$, $\left\langle X_{1}, X_{4}-X_{2}, X_{3}\right\rangle$, where $\alpha, \beta, \gamma, \varepsilon, \delta, \sigma$ are arbitrary real constants.

The application of the function invariant criterion under the group of Lie transformations allows to get an universal invariant of each subgroup from the obtained optimal system of subgroups in the space $R^{4}(u, h, \Phi, \Psi)$. The found universal invariants of all the subgroups allow to have the simplest samples of all essentially different (not linked with the point transformations) invariant solutions of the system (12). The non-degeneracy conditions of these solutions are obtained.

The variables $c_{k}(k=1,2, \ldots 10)$ in all the rest formulae are arbitrary real constants.

The system (12) has a trivial solution

$$
\Phi=\frac{c_{1}}{h}, \Psi=c_{2}
$$

which, if $c_{1} \neq 0$ because of (24) is a non-degenerate solution. Then the solution factorization with respect to solution (33) is carried out. In particular, all the invariant solutions of 0 rank are trivial.

\subsection{Invariant Solutions of 1 Rank}

The solutions which are invariant under the subgroups $\left\langle X_{2}+\alpha X_{4}\right\rangle,\left\langle X_{3}+\beta X_{4}\right\rangle$ are obtained.

7.1.1. The solution which is invariant under the subgroup $\left\langle X_{2}+\alpha X_{4}\right\rangle$ is a self-similar solution which is as follows 


$$
\Phi=u^{\alpha-1} F(\xi), \Psi=u^{\alpha-1} G(\xi), \xi=\frac{u^{2}}{4 h} .
$$

The substituting in the system (12) yields the factor system

$$
(\alpha-1) F(\xi)+2 \xi F^{\prime}(\xi)-4 \xi^{2} G^{\prime}(\xi)=0, F(\xi)+\alpha G(\xi)+\xi\left(2 G^{\prime}(\xi)-F^{\prime}(\xi)\right)=0,
$$

which reduces to Hein equation [14].

$$
\xi^{2}(\xi-1) F^{\prime \prime}(\xi)+\left(\frac{1}{2}-\alpha\right) \xi F^{\prime}(\xi)-\frac{1}{4}(\alpha-1)(\alpha-2) F(\xi)=0
$$

After the substituting

$$
F(\xi)=\xi^{\frac{1-\alpha}{2}} Q(\xi)
$$

this equation reduces to Gaussian differential equation

$$
\xi(\xi-1) Q^{\prime \prime}(\xi)+\left((1-\alpha) \xi-\frac{1}{2}\right) Q^{\prime}(\xi)+\frac{1}{4}\left(\alpha^{2}-1\right) Q(\xi)=0
$$

the solution of which is reflected [14] by means of hypergeometric function $F(a, b, c, \xi)$. As a result, the invariant solution under the subgroup $\left\langle X_{2}+\alpha X_{4}\right\rangle$ where $\alpha \neq 0$ is determined from the formulae

$$
\begin{gathered}
\Phi=h^{\frac{\alpha-1}{2}}\left(2 c_{3} F\left(\lambda, \lambda-1 ; \frac{1}{2} ; \frac{u^{2}}{4 h}\right)+c_{4} \frac{u}{\sqrt{h}} F\left(\mu, \mu-1 ; \frac{3}{2} ; \frac{u^{2}}{4 h}\right)\right), \\
\Psi=h^{\frac{\alpha}{2}}\left(c_{3}\left(1+\frac{1}{\alpha}\right) \frac{u}{\sqrt{h}}\left((\alpha-1)\left(\frac{u^{2}}{4 h}-4\right) F\left(\lambda, \lambda+1 ; \frac{3}{2} ; \frac{u^{2}}{4 h}\right)-F\left(\lambda, \lambda-1 ; \frac{1}{2} ; \frac{u^{2}}{4 h}\right)\right)+\right. \\
\left.+2 c_{4}\left(\frac{(\alpha-2) u^{2}}{12 h}\left(\frac{u^{2}}{4 h}-4\right) F\left(\mu, \mu+1 ; \frac{5}{2} ; \frac{u^{2}}{4 h}\right)-\left(\frac{u^{2}}{4 h}+\frac{4}{\alpha}\right) F\left(\mu, \mu-1 ; \frac{3}{2} ; \frac{u^{2}}{4 h}\right)\right)\right)
\end{gathered}
$$

Here $\lambda=\frac{1-\alpha}{2}, \mu=\frac{2-\alpha}{2}$. If $\alpha=2 k+1 \quad(k=0,1,2, \ldots)$ then hypergeometric series $F\left(\lambda, \lambda-1 ; \frac{1}{2} ; \frac{u^{2}}{4 h}\right)$, $F\left(\lambda, \lambda+1 ; \frac{3}{2} ; \frac{u^{2}}{4 h}\right)$, stop and if $\alpha=2 k \quad(k=0,1,2, \ldots)$ then hypergeometric series $F\left(\mu, \mu-1 ; \frac{1}{2} ; \frac{u^{2}}{4 h}\right)$, $F\left(\mu, \mu+1 ; \frac{3}{2} ; \frac{u^{2}}{4 h}\right)$, stop. Thus, if $c_{4}=0, \alpha=2 k+1(k=0,1,2, \ldots)$ and $c_{3}=0 \alpha=2 k(k=0,1,2, \ldots)$ then the solution determined from the formulae (34) is defined by means of elementary functions.

7.1.2. The solution which is invariant under subgroup $\left\langle X_{3}+\beta X_{4}\right\rangle$ has the following representation:

$$
\Phi=\exp (\beta u) F(h), \Psi=\exp (\beta u) G(h) .
$$

The substituting in the system (12) yields the factor system

$$
\beta F(h)+G^{\prime}(h)=0, F(h)+\beta G(h)+h F^{\prime}(h)=0,
$$

the general solution of which is reflected by means of Bessel function $J_{v}(\xi)$ and Neumann function $Y_{v}(\xi)$. The invariant solution is defined by the formulae 


$$
\Phi=\frac{1}{\sqrt{h}} \exp (\beta u)\left(c_{5} J_{1}(2 \beta \sqrt{h})+c_{6} Y_{1}(2 \beta \sqrt{h})\right), \quad \Psi=\exp (\beta u)\left(-c_{5} J_{0}(2 \beta \sqrt{h})+c_{6} Y_{0}(2 \beta \sqrt{h})\right)
$$

\subsection{Partially invariant solutions of 0 rank of defect 1 .}

Partially invariant $H$-solutions of 0 rank of defect 1 of the system (12) are partially invariant solutions for three-parameter subgroups $H \in\left\{\left\langle X_{1}, X_{2}, X_{4}\right\rangle,\left\langle X_{1}, X_{4}-X_{2}, X_{3}\right\rangle,\left\langle X_{2}, X_{3}, X_{4}\right\rangle\right\}$.

7.2.1. The equation of the least invariant manifold which has partially invariant $\left\langle X_{1}, X_{2}, X_{4}\right\rangle$-solution could be taken in the following form

$$
\Phi=\frac{\Psi}{\sqrt{h}} \operatorname{th} \theta,\left(\theta=\frac{1}{2} \ln \left(\frac{u-2 \sqrt{h}}{u+2 \sqrt{h}}\right)+c_{1}\right)
$$

The substituting in the system (12) reduces to the linear algebraic system of equations relative to $\Psi_{u}, \Psi_{h}$

$$
\Psi_{u} \operatorname{th} \theta+\Psi_{h} \sqrt{h}=-\frac{\theta_{u}}{\operatorname{ch}^{2} \theta} \Psi, \Psi_{u} \sqrt{h}+h \Psi_{h} \operatorname{th} \theta=-\left(\frac{h \theta_{u}}{\operatorname{ch}^{2} \theta}+\frac{\operatorname{th} \theta}{2}\right) \Psi
$$

where the matrix determinant is equal to $h\left(\operatorname{th}^{2} \theta-1\right) \neq 0$. This system is equivalent to the relations

$$
\Psi_{u}=\frac{\Psi}{\operatorname{th}^{2} \theta-1}\left(\frac{\operatorname{th} \theta}{2 \sqrt{h}}-\frac{u+2 \sqrt{h} \operatorname{th} \theta}{\left(u^{2}-4 h\right) \operatorname{ch}^{2} \theta}\right), \Psi_{h}=\frac{\Psi}{\left(\operatorname{th}^{2} \theta-1\right) \sqrt{h}}\left(\frac{u \operatorname{th} \theta+2 \sqrt{h}}{\left(u^{2}-4 h\right) \operatorname{ch}^{2} \theta}-\frac{\operatorname{th}^{2} \theta}{2 \sqrt{h}}\right) .
$$

The condition of consistency of which are as follows

$$
\left(\left(12 h-u^{2}\right) \operatorname{th}(2 \theta)-2 u \sqrt{h}\right) \Psi=0
$$

Therefore $\Psi=0, \Phi=0$.

7.2.2. The orbit of the partially invariant $\left\langle X_{1}, X_{4}-X_{2}, X_{3}\right\rangle$-solution is defined by the equation

$$
\Phi= \pm \frac{\sqrt{c_{7}+h \Psi^{2}}}{h}
$$

The result of substituting (36) in (12) is the system of equations

$$
\pm \frac{\Psi}{\sqrt{c_{7}+h \Psi^{2}}} \Psi_{u}+\Psi_{h}=0, \Psi_{u} \pm \frac{h \Psi}{\sqrt{c_{7}+h \Psi^{2}}} \Psi_{h}=\mp \frac{\Psi^{2}}{2 \sqrt{c_{7}+h \Psi^{2}}}
$$

The determinant of this system of linear algebraic equations with respect to $\Psi_{u}, \Psi_{h}$ is equal to $\frac{-c_{7}}{c_{7}+h \Psi^{2}}$. On the strength of the reduction theorem [9], the vanishing of the determinant is a necessary condition for irreducibility of a partially invariant solution (36) to an invariant solution.

If $c_{7}=0$, then the system (37) has a zero solution $\Psi=0$ only and due to (36), the function is $\Phi=0$.

If $c_{7} \neq 0$, then due to the system (37) it means

$$
\Psi_{u}=\mp \frac{\Psi^{2} \sqrt{c_{7}+h \Psi^{2}}}{2 c_{7}}, \Psi_{h}=\frac{\Psi^{3}}{2 c_{7}}
$$

The consistency condition for the system (38) is satisfied identically. According to the reduction theorem [9], due to (38), (12) it follows that the solution (36) is reduced to an invariant solution. Integrating the consistent system (38) and the use of the formula (36) yields 


$$
|\Psi|=\frac{2 \sqrt{c_{7}}}{\sqrt{\left(u+c_{8}\right)^{2}-4 h}},|\Phi|=\frac{\left|u+c_{2}\right| \sqrt{c_{7}}}{h \sqrt{\left(u+c_{8}\right)^{2}-4 h}}
$$

7.2.3. The least invariant manifold containing partially invariant $\left\langle X_{2}, X_{3}, X_{4}\right\rangle$-solution is determined by the equation

$$
\Phi=\frac{c_{1} \Psi}{\sqrt{h}}
$$

Similarly to the previous set, this solution is reduced to an invariant solution and is as follows $\Phi=0, \Psi=c_{2}$.

\subsection{Partially invariant solutions of 1 rank of defect 1 .}

For subgroups $H \in\left\{\left\langle X_{2}, X_{3}\right\rangle,\left\langle X_{2}+\gamma X_{3}, X_{4}\right\rangle,\left\langle X_{2}+X_{4}, X_{3}\right\rangle\right\}$ are established that partially invariant $H$ -solutions of the system (12) are reduced to its invariant $H^{\prime}$-solutions $\left(H^{\prime} \subset H\right)$. These invariant $H^{\prime}$-solutions are obtained. Simple wave is found for a homogeneous system, equivalent to system (12).

7.3.1. The equation of the least invariant manifold containing partially invariant $\left\langle X_{2}, X_{3}\right\rangle$-solution may be taken in the following form

$$
\Psi=f(\lambda), \lambda=h \Phi^{2}
$$

The substituting (40) in the system (12) yields

$$
\Phi_{u}+2 h \Phi f^{\prime}(\lambda) \Phi_{h}=-\Phi^{2} f^{\prime}(\lambda), 2 h \Phi f^{\prime}(\lambda) \Phi_{u}+h \Phi_{h}=-\Phi .
$$

Vanishing determinant of the linear system (41) is a necessary condition for irreducibility of this partially invariant solution to an invariant solution.

If the determinant of the linear system (41) is equal to zero, then $f(\lambda)= \pm \sqrt{\lambda}$ and a corresponding solution of the system (41) is $\Phi=0$, and due to (40), is $\Psi=c_{1}$.

If $f(\lambda) \neq \pm \sqrt{\lambda}$, then from the system (41) we have the following

$$
\Phi_{u}=\frac{f \Phi^{2}}{4 h f^{\prime 2} \Phi^{2}-1}, \Phi_{h}=-\frac{\left(2 h f^{\prime 2} \Phi^{2}-1\right) \Phi}{h\left(4 h f^{\prime 2} \Phi^{2}-1\right)} .
$$

The consistency condition for the system (42) is equality

$$
\lambda f^{\prime \prime}-2 \lambda f^{\prime 3}+f^{\prime}=0
$$

from which it follows that

$$
f(\lambda)=c_{9} \operatorname{arctg} \frac{\lambda-2 c_{9}^{2}}{\sqrt{4 c_{9}^{2} \lambda-\lambda^{2}}}+c_{10} .
$$

On the strength of the reduction theorem [9], from (42), (43), (12) it follows that the solution (40) is reduced to an invariant solution. The function $\Psi$ is as follows:

$$
\Psi=c_{9} \operatorname{arctg} \frac{h \Phi^{2}-2 c_{9}^{2}}{\Phi \sqrt{4 c_{9}^{2} h-h^{2} \Phi^{2}}}+c_{10},
$$

and the function $\Phi$ is determined from a completely integrable system (42), where the formula (43) is substituted in.

7.3.2. The least invariant manifold containing partially invariant $\left\langle X_{2}+\gamma X_{3}, X_{4}\right\rangle$-solution is defined by the equation

$$
\Phi=\frac{\Psi}{u+\gamma} f(\lambda), \lambda=\frac{(u+\gamma)^{2}}{h}
$$

The substituting (45) in the system (12) yields 


$$
f(\lambda) \Psi_{u}+(u+\gamma) \Psi_{h}=\left(\frac{f(\lambda)}{u+\gamma}-\frac{2(u+\gamma) f^{\prime}(\lambda)}{h}\right) \Psi,(u+\gamma) \Psi_{u}+h f(\lambda) \Psi_{h}=\left(\frac{(u+\gamma)^{2} f^{\prime}(\lambda)}{h}-f(\lambda)\right) \Psi
$$

Similarly to the previous solution it is stated that the equality $f(\lambda)= \pm \sqrt{\lambda}$ is a necessary condition for irreducibility [9] of this partially invariant solution to an invariant solution.

The solution of the system (46) where $f(\lambda)= \pm \sqrt{\lambda}$ is as follows $\Phi=\Psi=0$.

If $f(\lambda) \neq \pm \sqrt{\lambda}$, then it follows from the system (46) that

$$
\begin{aligned}
& \Psi_{u}=\frac{h(u+\gamma)^{2} f(\lambda)+h^{2} f^{2}(\lambda)-(u+\gamma)^{4} f^{\prime}(\lambda)-2 h(u+\gamma)^{2} f(\lambda) f^{\prime}(\lambda)}{h(u+\gamma)\left(h f^{2}(\lambda)-(u+\gamma)^{2}\right)} \Psi \\
& \Psi_{h}=-\frac{h f(\lambda)+h f^{2}(\lambda)-2(u+\gamma)^{2} f^{\prime}(\lambda)-(u+\gamma)^{2} f(\lambda) f^{\prime}(\lambda)}{h\left(h f^{2}-(u+\gamma)^{2}\right)} \Psi
\end{aligned}
$$

The system (47) is consistent if and only if function $f(\lambda)$ is a solution of the equation

$$
\begin{gathered}
\lambda\left(\lambda(\lambda-4)-4 f^{3}\left(f^{2}-\lambda\right)^{2}\right) f^{\prime \prime}-2 \lambda(\lambda-4)\left(f^{2}-\lambda\right)^{2} f f^{\prime 2}+2 \lambda f^{\prime}+ \\
+\left(f^{2}-\lambda\right)^{2}\left(3 f^{2}(\lambda-2)-2 \lambda\right) f f^{\prime}+\left(f^{2}-\lambda\right)^{2}\left(-f^{4}+\left(\lambda^{2}+1\right) f^{3}+2 f\right)=0 .
\end{gathered}
$$

On the strength of the reduction theorem [9], from (47), (48), (12) it follows that the solution (45) is reduced to an invariant solution. Function $\Psi$ is defined from a totally-integrable system (47), and function $\Phi$ is defined by the formula (45). In addition, function $f(\lambda)$ is a solution of the equation (48).

7.3.3. A general view of the orbit of a partially invariant $\left\langle X_{2}+X_{4}, X_{3}\right\rangle$-solution is

$$
\Phi=f(\lambda), \lambda=\frac{\Psi^{2}}{h} .
$$

The substituting (49) in the system (12) yields

$$
2 \Psi f^{\prime}(\lambda) \Psi_{u}+h \Psi_{h}=0, \Psi_{u}+2 \Psi f^{\prime}(\lambda) \Psi_{h}=\frac{\Psi^{2}}{h} f^{\prime}(\lambda)-f(\lambda)
$$

Due to (50), equality $f(\lambda)= \pm \sqrt{\lambda}$ is a necessary condition for irreducibility [9] of this partially invariant solution to an invariant solution.

Solution of system (50) for $f(\lambda)= \pm \sqrt{\lambda}$ has a form $\Phi=\Psi=0$.

If $f(\lambda) \neq \pm \sqrt{\lambda}$, then from the system (50) it yields the following

$$
\Psi_{u}=\frac{h f(\lambda)-f^{\prime}(\lambda) \Psi^{2}}{4 f^{\prime 2}(\lambda) \Psi^{2}-h}, \Psi_{h}=-\frac{2 f^{\prime}(\lambda)\left(h f(\lambda)-f^{\prime}(\lambda) \Psi^{2}\right) \Psi}{h\left(4 f^{\prime 2}(\lambda) \Psi^{2}-h\right)} .
$$

The system (51) is consistent if and only if function $f(\lambda)$ is a solution of the equation

$$
\left(4 \lambda f^{2}(\lambda)-\lambda^{2}\right) f^{\prime \prime}(\lambda)-2 \lambda^{2} f^{\prime 3}(\lambda)+2 f^{2}(\lambda) f^{\prime}(\lambda)=0 .
$$

On the strength of the reduction theorem [9], from (51), (52), (12) it follows that the solution (49) is reduced to an invariant solution for which $\Psi$ is defined from a totally-integrable system (51) and function $\Phi$ is defined by the formula (49). In addition, function $f(\lambda)$ satisfies the equation (52).

7.3.4. Simple wave. The system (12) is recorded by means of new unknown function $\Theta=h \Phi$ in the form of a homogeneous system

$$
\Theta_{u}+h \Psi_{h}=0, \Psi_{u}+\Theta_{h}=0
$$


The parametric representation of a simple wave of the system (53) is as follows:

$$
\Theta=\Theta(\lambda), \Psi=\Psi(\lambda)
$$

where $\lambda=\lambda(u, h)$ is a parameter of a simple wave. The substituting (54) in (53) generates the system of equations linking the unknown functions (54) and the required parameter $\lambda$ :

$$
\Theta^{\prime} \lambda_{u}+h \Psi^{\prime} \lambda_{h}=0, \Psi^{\prime} \lambda_{u}+\Theta^{\prime} \lambda_{h}=0
$$

where the prime denotes the derivative with respect to parameter $\lambda$. The condition for the existence of a nontrivial solution of the resulting linear algebraic system (55) with the $\lambda_{u}, \lambda_{h}$ unknown variables is as follows

$$
\Theta^{2}-h \Psi^{\prime 2}=0 \text {. }
$$

It follows a simple wave equation

$$
\Theta^{\prime}= \pm \sqrt{h} \Psi^{\prime},
$$

where $\Psi$ is any different from the constant continuously differentiable function of variable $\lambda$.

Due to (56), from the (55) system, it follows that the parameter of a simple wave is determined by the formula

$$
\lambda=u \mp 2 \sqrt{h}
$$

The corresponding solution of the system (12) is as follows

$$
\Phi=\frac{\Theta(u \mp 2 \sqrt{h})}{h}, \Psi=\Psi(u \mp 2 \sqrt{h}),
$$

where $\Psi$ is any continuously differentiable function and $\Theta$ is defined by the equation (56).

\section{Exact Non-Degenerate Solutions of the System of Equations of One-Dimensional Model of Shallow Water with a Straight Bottom}

Due to $\left(\Phi_{u}^{2}+h \Phi_{u}^{2}\right) \neq 0$, these non-degenerate exact solutions (33); (34); (35); (39); (42)-(44); (45), (47), (48); (49), (51), (52); (56) and (57) of the system (12) are the simplest significantly different (without reversible point transformations) exact solutions of the system. They depend on 13 arbitrary real constants $\alpha, \beta, \gamma, c_{1}, c_{2}, \ldots c_{10}(\alpha \neq 0)$, and the solution defined by the formulae (56), (57) has a functional arbitrary rule. The use of the (26)-(29) formulae of production of the system (12) solutions depending on 4 arbitrary real constants $b_{1}, b_{2}, b_{3}, b_{4} \quad\left(b_{2} b_{4} \neq 0\right)$, gives 17-parameter manifold with significantly different functional arbitrary rule of exact solutions of the system. The use of (30)-(32) infinitesimal formulae of the solutions production ("birth") generates infinite aggregate of exact solutions. The linear span of the aggregate forms an infinite-dimensional vector space $V$ of exact solutions of the system (12).

The formulae (23) of the total non-degenerate solutions of the system (3) of the shallow water equations, describing the propagation of surface waves above a horizontal bottom, generate infinite aggregate of exact solutions of the system when $(\Phi, \Psi)$ run through space $V$. The use of the formulae (16), (17) of solutions production generates additional non-degenerate exact solutions of the system (3).

The use of formulae (2) relative to infinite aggregate of exact solutions of the system (3) generates infinite aggregate of exact solutions of the system (1) of the shallow water equations describing the propagation of surface waves above a sloping bottom. The use of the formulae (18)-(22) of the solutions production ("birth") generates additional non-degenerate exact solutions of the system (1).

\section{Degenerate Solutions of the System of Equations of One-Dimensional Model of Shallow Water with a Straight Bottom}

\subsection{Degenerate Solutions of the System (3)}

The system (3) has a trivial degenerate solution: $u=$ const, $h=$ const. A non-trivial degenerate solution of the 
system (3) satisfies Jacobian vanishing $\frac{\partial(u, h)}{\partial(t, x)}=0$, which implies that $h=F(u)$, i.e. a non-trivial degenerate solution of the system (3) is its simple wave [9]. The substituting in (3) and the condition of non-trivial solutions generate a simple wave equation: $F^{\prime 2}=F$. Integration of this equation leads to two expressions for value $h$, the substituting of which in (3) generates the corresponding equations to determine $u$-parameter of a simple wave. The latter equations are Hopf equations of special types, the integration of which implicitly defines parameter $u$. As a result, many non-trivial degenerate solutions of system (3) reduce to the following solutions:

1) $h=0$ and function $u$ is defined implicitly by the equation

$$
f(u, x-t u)=0
$$

2) $h=\left(\frac{u}{2}+c\right)$, and functio $u$ is defined implicitly by the equation

$$
f\left(u, x-t\left(\frac{3 u}{2}+c\right)\right)=0
$$

where $c$ is an arbitrary real constant and $f$ is a nonconstant continuously differentiable function. The solutions (58), (59) are given in [7].

\subsection{Degenerate solutions of the system (1)}

where $k \neq 0$. The system (1) where $k \neq 0$ has a trivial degenerate solution $v=0, \eta=$ const. A non-trivial degenerate solution of the system (1) satisfies Jacobian vanishing $\frac{\partial(v, \eta)}{\partial(\tau, y)}=0$ from which it follows that

$$
\eta=F(v) .
$$

i.e. a non-trivial degenerate solution of the system (1) is its partially invariant solution of 1 rank and defect 1 .

The substituting (60) in (1) generates

$$
v_{\tau}+\left(v+F^{\prime}\right) v_{y}=0,\left(\left(F^{\prime}\right)^{2}-F-k y\right) v_{y}=k v .
$$

The consistency condition of the system (61) is an equality

$$
v F^{\prime \prime}+k F^{\prime}+(k+1) v=0,
$$

from which it follows that function $F$ is as follows

$$
\begin{aligned}
& F=-\frac{v^{2}}{2}+c_{1} v^{1-k}+c_{2}, \text { при } k \neq \pm 1 ; 0 ; \\
& F=-\frac{v^{2}}{2}+c_{1} \ln v+c_{2}, \text { при } k=1 ; \\
& F=c_{1} v^{2}+c_{2}, \text { при } k=-1 ;
\end{aligned}
$$

where $c_{1}, c_{2}$ are the arbitrary real constants.

The substituting (62)-(64) in (61) system and the integration of the equations generate the equations that define implicitly parametric function $v$

$$
\text { if } k \neq \pm 1 ; 0 ; \frac{1}{2} ; 2 \text { then: }
$$

$$
c_{1}\left((k-1) v^{1-k}+\frac{2 k-3}{k(k-2)} v^{2-k}+\frac{c_{1}(k-1)^{2}}{k(2 k-1)}\right)+c_{2} \frac{v}{k}+y v=c_{3} \text {, }
$$


if $k=1$ then:

$$
c_{1}\left(v \ln v+\frac{c_{1}}{v}-\tau\right)+c_{2} v-\frac{v^{3}}{2}+y v=c_{3},
$$

if $k=2$ then:

$$
c_{1}\left(\frac{\tau}{v}-\frac{1}{2} \ln v+\frac{c_{1}}{6 v^{3}}\right)+c_{2} \frac{v}{2}+y v=c_{3},
$$

if $k=\frac{1}{2}$ then:

$$
c_{1}\left(\frac{4}{3} v \sqrt{v} \frac{\tau}{v}-\frac{1}{2} \tau \sqrt{v}-\frac{c_{1}}{2} \ln v\right)+c_{2} v+y v=c_{3},
$$

if $k=-1$ then:

$$
v=v(y), c_{1}=-\frac{1}{2}, \frac{v^{3}}{2}+\left(y-c_{2}\right) v+c_{3}=0,
$$

where $c_{1}, c_{2}, c_{3}$ are the arbitrary real constants.

Thus, the set of non-trivial degenerate solutions of the system (1) where $k \neq 0$ reduces to the solutions (60) and (62)-(64) (excess of the free surface above the bottom) and parametric function $v$ (the propagation speed of surface waves) is implicitly defined by equations (65)-(69).

\section{Discussion and Conclusion}

One-parameter family of equations of one-dimensional model of shallow water describes the propagation of surface waves above a straight bottom is generated by the equations of one-dimensional model of shallow water with a horizontal bottom. The parameter of the family is the slope of the bottom. The main group of Lie transformations admitted by this system is determined by the system of linear differential equations. By means of the method of $A$-operators [9-13] it is found that the system of equations of one-dimensional model of shallow water with a straight bottom has an infinite aggregate of non-trivial zero-order conservation laws generated by the same system of linear differential equations. By the set of non-degenerate solutions the system of the equations of one-dimensional model of shallow water with a horizontal bottom becomes linear with the help of hodograph transformation, which is convenient to choose in accordance with the conservation laws of this system and its symmetry property.

Thus, this system of linear differential equations determines for the system of equations of one-dimensional model of shallow water with a straight bottom, the range of zero-order conservation laws, its main group of Lie transformations, and its set of non-degenerate exact solutions.

Infinite aggregate of non-degenerate solutions of one-dimensional model of shallow water with a straight bottom is obtained. All of the degenerate solutions of these equations are found. Thus, the data base of exact solutions of the equations of one-dimensional model of shallow water with a straight bottom is created. The solutions obtained in this paper may be used in the study of tsunami waves and fluid distribution in channels.

This work was supported by the Russian Foundation for Basic Research (project no. 12-01-00648), by the Program "Leading Scientific Schools" (project no. NSh 6706.2012.1), and by a grant from the Government of the Russian Federation for the State support of research teams headed by invited researchers (contract no. 12.740.11.1430).

\section{REFERENCES}

[1] J. J. Stoker. Water Waves: The Mathematical Theory with Applications, John Wiley and Sons, New York, 1958.

[2] E. N. Pelinovsky. "Hydrodynamics of tsunami waves, IAP RAS, Nizhny Novgorod, 1996, (in Russian). 
[3] G. B. Whitham. Linear and nonlinear waves, John Wiley and Sons, New York, 1974.

[4] G. F. Carrier, H. P. Greenspan. J. Fluid Mech., Vol. 4, 1958, 97-109.

[5] S. Yu. Dobrokhotov, B. Tirozzi. Localized solutions of a one-dimensional nonlinear system of shallow water equations with velocity , Russian Math. Surveys, 2010, Vol.65, No. 1, 177-179.

[6] S. Yu. Dobrokhotov, V. E. Nazaikinskii, B. Tirozzi. Asymptotic solution of the one-dimensional wave equation with localized initial data and with degenerating velocity. I, Russ. J. Math. Phys., 2010, Vol. 17, No. 4, 434-447.

[7] S. Yu. Dobrokhotov, S. B. Medvedev, D. S. Minenkov. On Replacements Reducing One-Dimensional Systems of Shallow-Water Equations to the Wave Equation with Sound Speed , Math. Notes, 2013, Vol. 93, No. 5, 704-714.

[8] P. J. Olver. Applications of Lie groups to differential equations, Springer-Verlag, New York, 1986.

[9] Yu. A. Chirkunov, S. V. Khabirov. Elements of symmetry analysis of differential equations of continuum mechanics, NSTU, Novosibirsk, 2012, (in Russian).

[10] Yu. A. Chirkunov. Method of A-operators and Conservation Laws for the Equations of Gas Dynamics, J. of Appl. Mech. and Techn. Phys., 2009, V.50, No. 2, $213-219$.

[11] Yu. A. Chirkunov. On Group Properties and Conservation Laws for Second-Order Quasi-Linear Differential Equations, J. of Appl. Mech. and Techn. Phys., 2009, V. 50, No. 3, 413 -418.

[12] Yu. A. Chirkunov. Conservation Laws and Group Properties of Equations of isentropic Gas Motion, J. of Appl. Mech. and Techn. Phys., 2010, V.51, No.1, $1-3$.

[13] Yu. A. Chirkunov. The Conservation Laws and Group Properties of the Equations of Gas Dynamics with Zero Velocity of Sound, J. of Appl. Math. and Mech., 2009, V. 73, No. 4, $421-425$.

[14] E. Kamke. Differential gleichungen losungsmethoden und losungen, Leipzig, 1959. 